\title{
From the Editors' Desk: Challenging Problems, Challenging Patients
}

\author{
Mitchell D. Feldman, MD, MPhil \\ Division of General Internal Medicine, Department of Medicine, University of California, San Francisco, San Francisco, CA, USA.
}

J Gen Intern Med 26(6):567

DOI: $10.1007 / \mathrm{s} 11606-011-1713-\mathrm{x}$

(c) Society of General Internal Medicine 2011

A ccording to the World Health Organization, depression affects more than 120 million persons worldwide and is a significant cause of disability. In fact, as assessed by disability adjusted life years (or DALYs), depression now ranks 2nd for men and women in the 15-44 age group. Along with other mental and behavioral disorders, it is also one of the most common problems seen in primary care, and is a frequent accompaniment to many chronic illnesses.

In this issue of JGIM, several papers examine depression and other mental/behavioral health disorders from a variety of perspectives. Sorkin et al. report on a study of the mental health needs and barriers to care among older Asian Americans in the US. They found that while expression of emotional distress and utilization of mental health services varied among the six Asian subgroups studied, several groups continue to underutilize care, possibly because of stigma and other cultural barriers. This study reminds us that our patients continue to confront more barriers to access quality care for mental and behavioral health conditions than for most of the other medical problems we manage.

Access to care does not guarantee satisfactory outcomes. Prior research has shown that many patients with depression continue to have significant symptoms despite an adequate trial of antidepressant medication. How to manage patients with 'treatment resistant depression' has been an ongoing challenge for clinicians and researchers. Trivedi et al. report on a systematic review of psychotherapy for patients with treatment resistant depression to determine if the evidence supports the use of psychotherapy to augment or substitute for drug therapy. They conclude that while there is insufficient evidence to draw any firm conclusions, psychotherapy (primarily cognitive therapy) is likely underutilized by primary care providers and should be considered as an option for patients with treatment resistant depression. Also in this issue, a study by Bayliss et al. reminds us that primary care patients with

Published online April 15, 2011 depression usually also have other medical co-morbidities that complicate their care. In contrast with some prior research, they found that incident depression did not worsen clinical outcomes in a cohort of patients with diabetes.

Patients with underlying mental disorders such as depression are often challenging for physicians to care for and are more likely to be perceived as "difficult". In this issue, Hinchey and Jackson present findings from a fascinating study that examined predictors and outcomes of difficult patient encounters in primary care. As they point out, most of the previous work in this area has focused on patient behavior. This study adds to our understanding of difficult encounters by more closely examining physician factors that are associated with difficult encounters and by looking at outcomes. They found that physicians with fewer years of clinical experience and those that rated lower on the Physician's Belief Scale (a measure of communication stylebetter scores imply a more open style) are more likely to perceive patient encounters as difficult. Patients involved in difficult encounters have less trust and lower satisfaction in their physicians and are more likely to have worsening of their symptoms. Improving physician communication skills should be a priority at all levels of medical education.

And speaking of education, June marks the time that many of our trainees (students, residents and fellows) prepare to move on to the next stage of their career, while newly admitted medical students enjoy their last unfettered summer. In this issue, our Healing Arts section features a "White Coat" address delivered by Dr. Bernard Karnath to the incoming medical school class at the University of Texas Medical Branch at Galveston. He tells them that as they are symbolically welcomed into the profession of medicine by donning a white coat for the first time, that they are now bound to the professional commitments of altruism, responsibility, respect and compassion. These are important virtues for all of us to strive for as we approach the next academic year.

Corresponding Author: Mitchell D. Feldman, MD, MPhil; Division of General Internal Medicine, Department of Medicine, University of California, San Francisco, 400 Parnassus Avenue, San Francisco, CA 94143-0320, USA (e-mail: mfeldman@medicine.ucsf.edu). 